\title{
Bariatric Surgery and COVID-19: What We Have Learned from the Pandemic in Iran: a Retrospective Observational Cohort Study
}

\author{
Gholamreza Moradpour ${ }^{1} \cdot$ Masoud Amini $^{1} \cdot$ Nader Moeinvaziri $^{1} \cdot$ Seyed Vahid Hosseini ${ }^{1} \cdot$ Shirin Rajabi $^{1}$. \\ Cain C. T. Clark ${ }^{2} \cdot$ Babak Hosseini $^{1} \cdot$ Leila Vafa $^{1} \cdot$ Neda Haghighat $^{1}$ (i)
}

Received: 5 April 2021 / Revised: 10 October 2021 / Accepted: 18 October 2021 / Published online: 30 October 2021

(c) The Author(s), under exclusive licence to Springer Science+Business Media, LLC, part of Springer Nature 2021

\begin{abstract}
Purpose Little is known about the symptoms of coronavirus disease 2019 (COVID-19) on patients with morbid obesity following bariatric surgery (BS) in Iran. Thus, we sought to investigate the symptoms and effect of COVID-19 in patients with morbid obesity following, or candidates for, BS in Iran.

Materials and Methods In this retrospective observational cohort study, we enrolled 236 morbid obese patients following (surgical group) or candidates (nonsurgical group) for bariatric surgery. Demographics, probable COVID-19 incidence, acute and persistent COVID-19 symptoms, and clinical outcome parameters of bariatric patients and candidates for BS were compared. The incidence of probable COVID-19 was assessed including the clinical definition of probable case, according to World Health Organization criteria.

Results The incidence of probable COVID-19 among surgical and nonsurgical groups was significantly different (20.6\% vs $26.08 \%$, respectively, $p=0.046$ ). The probable case of surgical patients had a shorter length of symptoms and hospitalization duration, and a lower proportion of admission in ICUs and hospitals with respect to nonsurgical patients $(p<0.001)$. Surgical patients had a greater prevalence of persistent symptoms including anorexia, food intolerance, and anosmia-hyposmia than nonsurgical patients. Moreover, surgical patients with probable COVID-19 had a significantly higher proportion of diabetic patients than surgical patients without probable COVID-19 (20\% vs 9.3\%).

Conclusion These findings highlight the need to evaluate the persistent symptoms of COVID-19 and the importance of nutritional support for at least several weeks after COVID-19 symptom onset. Moreover, it seems that COVID-19 incidence in post-bariatric surgery patients could reduce the effectiveness of bariatric surgery in the resolution of diabetes.
\end{abstract}

Keywords Bariatric surgery $\cdot$ COVID-19 $\cdot$ Long COVID $\cdot$ Prolonged symptoms $\cdot$ Acute symptoms $\cdot$ Weight loss $\cdot$ Obesity

Key Points Key Point 1

- The incidence of probable COVID-19 among surgical and nonsurgical groups was significantly different (20.6\% vs $26.08 \%)$. Key Point 2

- Surgical patients had a greater prevalence of anorexia, food intolerance, and anosmia-hyposmia than nonsurgical patients.

Key Point 3

- Surgical patients with probable COVID-19 status had a significantly higher proportion of diabetic patients than surgical patients without probable COVID-19 (20\% vs 9.3\%).

Neda Haghighat

neda.hag@gmail.com

1 laparoscopy Research Center, Shiraz University of Medical Sciences, Shiraz, Iran

2 Centre for Intelligent Healthcare, Coventry University, Coventry CV1 5FB, UK

\section{Introduction}

The coronavirus disease 2019 (COVID-19), caused by severe acute respiratory syndrome coronavirus 2 (SARS-CoV-2), was announced as a global pandemic by the World Health Organization (WHO) on 11 March 2020. Overwhelming evidence suggests that COVID-19 patients with obesity and other comorbidities, including metabolic syndrome, are at a higher risk of requiring intensive care unit (ICU) stay and have increased rates of mortality [1]. Obesity is associated with a weak pulmonary capacity (decreased expiratory reserve volume, functional capacity, and lung compliance) and a rise in inflammation, which may increase the morbidity of COVID-19 infections [2]. Bariatric surgery can elicit a substantial and sustained weight loss, an improvement in comorbidities and amelioration of the 
obesity-associated proinflammatory state, and survival benefits in patients with morbid obesity [3, 4]. On the other hand, COVID-19 may be associated with clinical manifestations, ranging from alterations in smell and taste to severe respiratory distress requiring intensive care that might be related with weight loss and malnutrition in patients. However, in patients with severe COVID-19, cytokine storm, the use of mechanical ventilation, and the prolonged hospital stays could further increase the risk of malnutrition [5, 6]. Although, patients with mild COVID-19 undergoing home management might also suffer from malnutrition due to alterations of smell and taste, fatigue, and lack of appetite that are common symptoms in COVID-19 patients [7]. Disease-associated decreases in food intake and malnutrition can contribute to tissue wasting; moreover, confinement at home and COVID-19 symptoms may concurrently limit engagement in physical activity, leading to loss of lean mass [8]. These factors might contribute to malnutrition even in non-hospitalized patients. Recently, in Italy, a high incidence area, similar to Iran, it has been shown that COVID-19 might negatively affect body weight and nutritional status [9].

Recent evidence has revealed that a range of persistent symptoms can remain long after the acute SARS-CoV-2 infection, and this condition is now named "long COVID" by recognized research institutes [10]. The National Institutes of Health (NIH) uses the US Centers for Disease Control and Prevention (CDC) definition of long COVID, which describes the condition as sequelae that extend beyond 4 weeks after initial symptoms [11]. Studies have shown that long COVID can affect the whole spectrum of people with COVID-19, from those with the very mild acute disease to the most severe forms. Like acute COVID-19, long COVID can involve multiple organs and can affect many systems including, but not limited to, the respiratory, cardiovascular, neurological, gastrointestinal, and musculoskeletal systems [10].

Despite reports of symptoms resulting from COVID-19 in patients following bariatric surgery, there is no literature describing persistent symptoms in bariatric patients and the studies of persistent symptoms in general population have focused on severe COVID-19 patients. Data suggest that about one-third of patients continued to exhibit symptoms more than 4 weeks after symptom onset [12]. Therefore, given the current dearth of evidence, we sought to investigate the incidence of weight loss, and the acute and persistent effect of COVID-19 in patients with morbid obesity following, or candidates for, BS in Iran, a high incidence area.

\section{Materials and Methods}

\section{Design and Setting of the Study}

The present study is a retrospective observational cohort study of 236 morbid obese patients undergoing or candidate for bariatric surgery at Ghadir Mother and Child Hospital, Shiraz, Iran. Ethical approval was obtained from the Ethics Committee of Shiraz University of Medical Sciences (IR. SUMS.REC.1400.402).

\section{Study Population}

\section{Selection Criteria}

We included adult patients, aged 18 years and over, with morbid obesity (BMI was $>40$ or $>35$ with comorbidities) following bariatric surgery that matched to the control group, who were morbid obese candidate for bariatric surgery. Among surgical patients, only subjects that had undergone the procedure between 12 and 18 months before the study were considered for the analysis; this is considered the minimum time to weight loss stabilization and remission of comorbidities [13]. We excluded the patients with severe hepatic and renal impairments, cardiovascular and cerebrovascular diseases, and chronic pulmonary diseases such as asthma. Meanwhile, women who were pregnant or lactating were also excluded.

\section{Data Collection During Lockdown}

All patients were contacted between November 2020 and March 2021. The patients were requested to answer the questions including their demographic data, bariatric surgery history, COVID-19 symptoms, weight loss, comorbidities, any hospital admission, length of hospital stay, ICU admission, and death. Demographic data included age, sex, weight, height, body mass index, and type of bariatric surgery. The most recent biochemical and hematological test results were acquired. Because the present study is performed in a highrisk transmission region from February 2020, in the epidemic peak, the shortage of diagnostic tools led to a high rate of undiagnosed patients [14]; therefore, in addition to the confirmed COVID-19 patients, the present study mainly focused on clinically suspected COVID-19 cases, the subjects meeting the WHO clinical criteria for "probable cases." The patients with an episode of anosmia/dysgeusia or had a chest imaging suggestive of COVID-19 or the association of rapid onset fever (self-measured temperature $\geq 37.5^{\circ} \mathrm{C}$ ) and cough or the association of any three or more of the following signs and symptoms: fever, cough, general weakness, headache, myalgia, sore throat, nose discharge/swelling, nausea/vomiting, or diarrhea were considered probable cases or clinically suspected COVID-19 $[15,16]$. This study included patients that reported symptoms or outcomes assessed at 4 or more weeks post COVID-19 onset. Moreover, among participants in this study, 25 patients in the 
surgical patient group and 29 patients in the nonsurgical group had a confirmed or clinically suspected COVID-19, yielding a probable COVID-19 status that 17 of 25 cases in the surgical group and 19 of 30 cases in the nonsurgical group were confirmed cases based on nasopharyngeal swab test, chest X-rays, or chest CT scans.

\section{Statistical Analysis}

Continuous variables were expressed as mean (SD), while categorical variables were expressed as absolute values and percentages. Independent samples $t$-tests for continuous variables and Pearson's $\chi^{2}$ test or Fisher's exact test for categorical variables were used to compare characteristics of patients according to their probable COVID-19 status. Statistical significance was set, a priori, at $p<0.05$.

\section{Results}

\section{Characteristics of Patients According to Surgical or Nonsurgical}

Of 188 morbid obese patients following BS that their data were recorded in the national obesity surgery database, 67 were not included in the present study: 34 patients refused to participate, 12 followed in other health areas, and 21 patients were lost to follow-up. Finally, 121 patients following BS were included in the surgical group that were matched based on sex and age to 115 morbid obese patient candidates for BS (nonsurgical group). The mean BMI was $29.65 \pm 6.2$ and $45.08 \pm 5.8 \mathrm{~kg} / \mathrm{m}^{2}$ and the mean age was $45.3 \pm 11.3$ and $45.1 \pm 10.1$ years in the surgical and nonsurgical groups, respectively. The main procedures performed were Rouxen-Y gastric bypass (54.4\%) and sleeve gastrectomy (45.0\%), while the mean time elapsed between surgery and the time of the call was 15 months. As a predictable effect for BS, surgical patients presented a significantly lower BMI (30.7 vs $43.5, p<0.001)$ and lower incidence of main comorbidities, such as obstructive sleep apnea syndrome, hypertension, diabetes, and hyperlipidemia. Patient characteristics are summarized in Table 1.

\section{The COVID-19 Acute and Persistent Symptoms}

The COVID-19 symptoms as mentioned for probable cases were detected in $25(20.6 \%)$ patients in the surgical group and $30(26.0 \%)$ patients in the nonsurgical group, with a statistically significant difference $(p=0.046)$. Moreover, the rate of confirmed cases was respectively $14.04 \%$ in surgical patients and $16.52 \%$ in the nonsurgical group. Among probable cases, surgical patients had a greater prevalence of anorexia (13.2\%) and food intolerance
Table 1 Characteristics of the cohort surgical and nonsurgical patients

\begin{tabular}{|c|c|c|c|}
\hline Variable & $\begin{array}{l}\text { Surgical } \\
\text { group, } \\
N=121\end{array}$ & $\begin{array}{l}\text { Nonsurgi- } \\
\text { cal group, } \\
N=115\end{array}$ & $p$ value \\
\hline Age, years & $45.3 \pm 11.3$ & $45.1 \pm 10.1$ & 0.49 \\
\hline Sex (male), $n(\%)$ & $6(24.1)$ & $7(23.5)$ & 0.33 \\
\hline Weight, kg & $80.8 \pm 16.7$ & $122.4 \pm 25.1$ & $<0.001$ \\
\hline Height, cm & $165.1 \pm 9.4$ & $164.8 \pm 12.5$ & 0.65 \\
\hline BMI, $\mathrm{kg} / \mathrm{m}^{2}$ & $29.65 \pm 6.2$ & $45.08 \pm 5.8$ & $<0.001$ \\
\hline \multicolumn{4}{|l|}{ Type of surgery, $n(\%)$} \\
\hline RYGB & $66(54.5)$ & - & \\
\hline SG & $55(45.5)$ & - & \\
\hline $\begin{array}{l}\text { Obstructive sleep apnea, } \\
n(\%)\end{array}$ & $20(16.5)$ & $27(23.5)$ & 0.002 \\
\hline Type 2 diabetes, $n(\%)$ & $13(10.7)$ & $32(27.8)$ & $<0.001$ \\
\hline Hypertension, $n(\%)$ & $26(21.3)$ & $40(34.7)$ & 0.001 \\
\hline Hyperlipidemia, $n(\%)$ & $20(16.4)$ & $48(41.7)$ & $<0.001$ \\
\hline
\end{tabular}

All results are presented as the mean $\pm \mathrm{SD}$ or number (percentage) as appropriate. $B M I$, body mass index; $R Y G B$, Roux-en-Y gastric bypass; $S G$, sleeve gastrectomy; $p$ value, refer to compare group surgical and nonsurgical

(4.1\%) and less myalgia (12.3\%), headache $(14.8 \%)$, and cough $(5.7 \%)$ than nonsurgical patients $(7.8 \%, 1.7 \%$, $21.7 \%, 19.1 \%$, and $10.5 \%$ respectively). Moreover, among probable cases, the length of symptoms was significantly lower in the surgical group than in the nonsurgical group $(7.8 \pm 9.4$ vs $10.1 \pm 8.8, p<0.001)$. Among the probable COVID-19 surgical group, 4 (3.3\%) required hospitalization and $1(0.8 \%)$ ICU admission was reported, which was significantly lower than the probable COVID-19 nonsurgical group (9 (7.8\%), 3 (2.6\%), $p<0.001$ respectively). Moreover, the length of hospital stay was lower in the surgical group than in the nonsurgical group (hospital stay, $6.2 \pm 8.8$ vs $10.4 \pm 7.9$ days, $p<0.001)$. Importantly, among probable cases, surgical patients had a higher rate of weight loss during COVID-19 infection than nonsurgical patients (Table 2). The most frequent persistent symptoms were fatigue, dyspnea, and ageusia-dysgeusia. Among probable cases, surgical patients had a greater prevalence of anorexia (6.6\%), food intolerance (3.3\%), and anosmia-hyposmia (5.7\%) and less tachycardia (0.8\%) and chest pain $(0 \%)$ than nonsurgical patients $(3.4 \%, 0 \%$, $2.6 \%, 3.4 \%$, and $2.6 \%$ respectively) (Table 3 ).

\section{Characteristics of the Surgical Patients According to Clinically Probable COVID-19}

Among patients post-bariatric surgeries, there was no difference regarding the type of surgery, time elapsed since 
Table 2 COVID-19-related acute symptoms and outcomes according to surgical and nonsurgical groups in probable COVID-19 patients

\begin{tabular}{|c|c|c|c|}
\hline Variable & $\begin{array}{l}\text { Surgical group, } n=121 \\
\text { Probable cases, } n=25\end{array}$ & $\begin{array}{l}\text { Nonsurgical group, } n=115 \\
\text { Probable cases, } n=30\end{array}$ & $p$ value \\
\hline Probable cases & $25(20.6)$ & $30(26.08)$ & 0.046 \\
\hline Confirmed cases & $17(14.04)$ & $19(16.52)$ & 0.333 \\
\hline Any symptoms & $28(23.1)$ & $33(28.6)$ & 0.048 \\
\hline Fever $\left(>37.5^{\circ} \mathrm{C}\right)$ & $19(15.7)$ & $20(17.3)$ & 0.091 \\
\hline Shivering & $11(9.9)$ & $14(12.1)$ & 0.291 \\
\hline Cough & $7(5.7)$ & $12(10.5)$ & 0.048 \\
\hline Anorexia & $16(13.2)$ & $9(7.8)$ & 0.045 \\
\hline Anosmia & $14(11.5)$ & $12(10.4)$ & 0.442 \\
\hline Food intolerance & $5(4.1)$ & $2(1.7)$ & 0.002 \\
\hline Running nose & $19(15.7)$ & $20(17.3)$ & 0.650 \\
\hline Nausea/vomiting & $10(8.2)$ & $6(5.2)$ & 0.08 \\
\hline Diarrhea & $6(4.9)$ & $6(5.2)$ & 0.11 \\
\hline Ageusia/dysgeusia & $11(9.0)$ & $10(8.6)$ & 0.74 \\
\hline Asthenia & $19(15.7)$ & $25(21.7)$ & 0.048 \\
\hline Myalgia & $15(12.3)$ & $25(21.7)$ & 0.011 \\
\hline Headache & $18(14.8)$ & $22(19.1)$ & 0.027 \\
\hline Sore throat & $20(16.5)$ & $21(18.2)$ & 0.15 \\
\hline Weight loss during COVID-19 infection (\%) & 13.75 & 7.37 & 0.03 \\
\hline Length of symptoms (days; average \pm SD) & $7.8 \pm 9.4$ & $10.1 \pm 8.8$ & 0.02 \\
\hline \multicolumn{4}{|l|}{ Outcomes } \\
\hline Hospital admission & $4(3.3)$ & $9(7.8)$ & $<0.001$ \\
\hline ICU admission & $1(0.8)$ & $3(2.6)$ & $<0.001$ \\
\hline Death & 0 & 0 & - \\
\hline Hospital stay (days; average \pm SD) & $6.2 \pm 8.8$ & $10.4 \pm 7.9$ & $<0.001$ \\
\hline
\end{tabular}

All results are presented as the mean $\pm \mathrm{SD}$ or number (percentage) as appropriate

Table 3 COVID-19-related persistent symptoms and outcomes according to surgical and nonsurgical groups in probable COVID-19 patients

\begin{tabular}{llll}
\hline Variable & $\begin{array}{l}\text { Surgical group, } n=121 \\
\text { Probable cases, } n=25\end{array}$ & $\begin{array}{l}\text { Nonsurgical group, } n=115 \\
\text { Probable cases, } n=30\end{array}$ & $p$ value \\
\hline Probable cases & $25(20.6)$ & $30(26.08)$ & 0.046 \\
Confirmed cases & $17(14.04)$ & $19(16.52)$ & 0.333 \\
Any symptoms & $13(10.7)$ & $14(12.1)$ & 0.091 \\
Anorexia & $8(6.6)$ & $4(3.4)$ & 0.038 \\
Anosmia/hyposmic & $7(5.7)$ & $3(2.6)$ & 0.015 \\
Food intolerance & $4(3.3)$ & 0 & - \\
Fatigue & $9(7.4)$ & $9(7.8)$ & 0.975 \\
Diarrhea & $1(4)$ & 0 & - \\
Ageusia/dysgeusia & $9(7.4)$ & $6(5.2)$ & 0.051 \\
Dyspnea & $7(5.7)$ & $10(8.6)$ & 0.288 \\
Myalgia & $2(1.6)$ & $3(2.6)$ & 0.12 \\
Headache & $1(0.8)$ & $2(1.7)$ & 0.450 \\
Cough & $5(4.1)$ & $8(6.9)$ & 0.066 \\
Joint pain & 0 & $1(0.7)$ & - \\
Tachycardia & $1(0.8)$ & $4(3.4)$ & 0.045 \\
Chest pain & 0 & $3(2.6)$ & - \\
Dizziness & $1(0.8)$ & $1(0.7)$ & 0.88 \\
\hline
\end{tabular}

All results are presented as the number (percentage) as appropriate 
surgery, or baseline characteristics between probable COVID-19 patients and not probable COVID-19 patients. However, surgical patients with probable COVID-19 status had a significantly higher proportion of patients with persistent T2D (20\% vs 9.3\%, $p=0.004)$ and a non-significantly higher proportion of patients with HTN (24\% vs $20.83 \%, p=0.08)$ and lower serum zinc $(75.58 \pm 8.21$ vs $86.97 \pm 32.21, p=0.08)$, than not probable COVID-19 surgical patients (Table 4).

\section{Discussion}

The present study investigated the impact of the COVID19 infection on symptoms of patients with morbid obesity, following or candidate for bariatric surgery. This is the first study that reported the rates of persistent symptoms consistent with COVID-19 in surgical and nonsurgical patients. Our data suggest that surgical patients present significantly higher weight loss during acute phase of COVID-19 than nonsurgical patients. Moreover, interestingly, we noted that among surgical patients, those with probable COVID-19 had a higher incidence of diabetes than the patients without probable COVID-19. It seems that COVID-19 incidence in post-bariatric surgery patients could reduce the effectiveness of bariatric surgery in the resolution of T2DM. However, this is the first study to have reported this finding and more studies are needed to discern causality. Proinflammatory state, attenuation of the innate immune response, and possibly increased level of ACE2, along with vascular dysfunction and prothrombotic state in people with diabetes probably contribute to higher susceptibility for SARS-CoV-2 infection and worsened prognosis. On the other hand, activated inflammation, islet damage induced by a virus infection, and treatment with glucocorticoids could, in turn, result in impaired glucose regulation in diabetic patients, thus working as an amplification loop to aggravate the disease. Therefore, glycemic management in people with COVID-19 is important [17].

As suggested by the European Society of Enteral and Parenteral Nutrition (ESPEN), prevention, diagnosis, and treatment of malnutrition should be considered in the management of COVID-19 patients to improve the prognosis [6]. There are few studies currently available that have assessed the general, or selected, populations, such as elderly or critically ill patients $[18,19]$; however, of the available evidence, studies support the hypothesis that COVID-19 can lead to undesirable weight loss and malnutrition in patients with morbid obesity following BS.

In our study, we included patients with mild disease severity, managed at home, to patients with high disease severity admitted to a clinical setting. We observed that weight loss occurred in all patients in a relatively short time (mean disease duration: 8.95 days). Recently, Filippo et al. reported that the length of stay in hospital was significant and independent predictor of weight loss, reflecting the importance of disease severity and inflammation to weight loss [9]. However, some previous studies showed that even
Table 4 Characteristics of the surgical patient group according to probable COVID-19

\begin{tabular}{llll}
\hline Variables & Probable COVID-19 & Not probable COVID-19 & $p$ \\
& $N=25$ & $N=96$ & \\
\hline Age, years & $45.3 \pm 11.3$ & $44.7 \pm 10.6$ & 0.45 \\
Sex (male), $n(\%)$ & $6(24.1)$ & $16(17.8)$ & 0.12 \\
Weight, kg & $80.8 \pm 16.7$ & $82.4 \pm 19.1$ & 0.44 \\
Height, cm & $165.1 \pm 9.4$ & $166.5 \pm 8.9$ & 0.78 \\
BMI, kg/m ${ }^{2}$ & $29.65 \pm 6.2$ & $29.7 \pm 5.6$ & 0.45 \\
Excess weight loss, $\%$ & $64.5 \pm 16.6$ & $62.6 \pm 18.3$ & 0.78 \\
Type of surgery, $n(\%)$ & & & \\
RYGB & $13(52.0)$ & $51(53.1)$ & 0.77 \\
SG & $12(48.0)$ & $45(46.9)$ & 0.84 \\
Obstructive sleep apnea syn- & $4(16)$ & $13(13.5)$ & 0.22 \\
$\quad$ drome, $n(\%)$ & & & 0.004 \\
Type 2 diabetes, $n(\%)$ & $4(20)$ & $9(9.3)$ & 0.08 \\
Hypertension, $n(\%)$ & $6(24)$ & $20(20.83)$ & 0.75 \\
Hyperlipidemia, $n(\%)$ & $4(16)$ & $16(16.6)$ & 0.77 \\
Vitamin D, ng/mL & $24.44 \pm 8.2$ & $26.2 \pm 18.01$ & 0.08 \\
Zinc, $\mu \mathrm{g} / \mathrm{dL}$ & $75.58 \pm 8.21$ & $86.97 \pm 32.21$ & 0.29 \\
Hb, mg/dL & $12.9 \pm 3.6$ & $12.6 \pm 2.4$ & \\
\hline
\end{tabular}

All results are presented as the mean $\pm \mathrm{SD}$ or number (percentage) as appropriate. BMI, body mass index; $R Y G B$, Roux-en-Y gastric bypass; $S G$, sleeve gastrectomy; $H b$, hemoglobin; $p$ value, refer to compare group probable COVID-19 and not probable COVID-19 
short periods of bed rest can induce marked reductions in muscle protein synthesis, resulting in loss of skeletal muscle mass [20-22], which is strongly associated with malnutrition. It is noteworthy that COVID-19 probable patients had a weight loss $>5 \%$ of their body weight in their COVID19 disease period, the threshold used to diagnose cancer cachexia [23]. Several mechanisms may contribute to weight loss and malnutrition in COVID-19 patients; for instance, acute systemic inflammation strongly affects several metabolic [24] and hypothalamic [25] pathways contributing to anorexia and decreased food intake as well as elevation of resting energy expenditure and increased muscle catabolism [26]. Acute inflammatory environments can trigger persistent neuroinflammatory responses in exposed individuals, which may prolong inflammation and wasting, even after the acute phase [25, 27]. Moreover, alterations of smell and taste, as well as fatigue and lack of appetite, in patients with mild COVID-19, could deleteriously affect food intake [7]. The primary results of this study also showed that bariatric patients had a higher incidence of anorexia and food intolerance than the control group, which contributed to greater weight loss during SARS-CoV-2 infection. In addition, this study reported a higher rate of persistent symptoms related to food intake including food intolerance, anorexia, anosmia, ageusia, and asthenia in surgical patients. The United Kingdom Office for National Statistics estimated the 5-week prevalence of loss of smell and loss of taste as $7.9 \%$ and $8.2 \%$ of all people who have had COVID-19, respectively [28]. Other studies have found varying prevalence of olfactory and gustatory dysfunction, ranging from 11 to $45.1 \%$ of cohorts of patients who have recovered from acute COVID19 [29-31]. Since SARS-CoV-2 can affect neuronal cells by both direct and indirect mechanisms, this can lead to various neurological manifestations also including anosmia and hypogeusia. Anosmia and hypogeusia are present both in mild-to-severe cases of COVID-19 and many studies which evaluated olfactory and gustatory dysfunctions in mild-tosevere COVID-19 patients confirmed persistent loss of smell up to 6 months after symptom onset [12].

On the other hand, malnutrition is known to be associated with the risk for viral pneumonia, as far back as the 1918 influenza pandemic [32]. In addition, Bel Lassen et al. reported that persistent T2D and lower BMI after BS might be associated with the risk and the severity of COVID-19 [33]. Thus, assessment and monitoring of the patients with SARS-CoV-2 infection following bariatric surgery in the COVID-19 pandemic must be carefully performed.

In the present study, among patients with COVID-19 probable status, patient candidates for BS had a longer duration of symptoms $(7.8 \pm 9.4$ vs $10.1 \pm 8.8, p<0.001)$ and hospitalization $(6.2 \pm 8.8$ vs $10.4 \pm 7.9, p<0.001)$, compared to the patients who underwent bariatric procedures. Moreover, candidates for BS had a higher prevalence of hospital
(30\% vs $16 \%, p<0.001)$ or ICU $(10 \%$ vs $4 \%, p<0.001)$ admission. Similar to this study, Marchesi et al. reported a statistically significant difference in the rates of hospitalization $(0.3 \%$ vs $2.4 \%, p=0.02)$ among the bariatric patients and the candidate groups [16].

The findings of the present study also showed that the bariatric patients had a lower incidence of weight and metabolic abnormalities, including type 2 diabetes and hypertension, which may have contributed to better outcomes in patients with SARS-CoV-2 infection. However, our results are consistent with two systematic reviews and meta-analyses that reported higher rates of hospitalization, ICU admission, severe disease, and the need for invasive mechanical ventilation, in patients with obesity who develop COVID-19 $[34,35]$. Therefore, a reduction in excessive adipose tissue after bariatric surgery may have resulted in an improvement in the inflammatory response, enhancing immunity, and cardiometabolic risk factors, thereby contributing to a lesser severity of COVID-19 infection [36]. Indeed, our results point to the importance of weight management in patients with morbid obesity. Post-bariatric surgery, nutritional evaluation and intervention are essential for the prevention of nutritional deficiency, not only for enhancing the immune function but also for preventing severe weight loss in patients with COVID-19 infection, irrespective of those adhering to home management or hospitalization. Therefore, we suggest careful reassessment is needed to monitor weight changes and nutritional status in patients following bariatric surgery with symptomatic COVID-19.

\section{Limitations}

Several limitations must be considered in the interpretation of our results. For instance, due to the cross-sectional and observational design, not causal inferences can be made. Therefore, a well-designed cohort or randomized clinical trial study is needed to determine the extent to which malnutrition contributes to a higher incidence of COVID-19. Further limitations of our study are the use of patient-reported symptoms and weight loss during their COVID-19 disease period that may be underestimated.

\section{Conclusion}

In conclusion, we reported a high rate of weight loss and risk of malnutrition among bariatric patients with COVID19 probable status. Moreover, we observed that COVID19 probable cases submitted to a bariatric procedure had a shorter length of symptoms and hospitalization duration, and a lower proportion of admission in ICUs and hospitals with respect to non-operated patients. Furthermore, the high ratio of diabetic patients in surgical COVID-19 patients showed 
that COVID-19 incidence in post-bariatric surgery patients could reduce the effectiveness of bariatric surgery in the resolution of T2DM. Therefore, bariatric surgery might yield a favorable effect in patients with morbid obesity; however, nutritional screening strategies should be carefully implemented and monitored.

\section{Declarations}

Ethics Approval All procedures performed in studies involving human participants were in accordance with the ethical standards of the institutional and/or national research committee and with the 1964 Helsinki Declaration and its later amendments or comparable ethical standards.

Informed Consent Informed consent was obtained from all individual participants included in the study.

Conflict of Interest The authors declare no competing interests.

\section{References}

1. Dietz W, Santos-Burgoa C. Obesity and its implications for COVID-19 Mortality. Obesity. 2020.

2. Capelo AV, da Fonseca VM, Peixoto MVM, et al. Visceral adiposity is associated with cytokines and decrease in lung function in women with persistent asthma. Rev Port Pneumol (English Ed.). 2016. https://doi.org/10.1016/j.rppnen.2016.02.005.

3. Yeo C, Kaushal S, Lim B, et al. Impact of bariatric surgery on serum uric acid levels and the incidence of gout—a meta-analysis. Obes Rev. 2019. https://doi.org/10.1111/obr.12940.

4. Yeo D, Yeo C, Low TY, et al. Outcomes after metabolic surgery in Asians-a meta-analysis. Obes Surg. 2019. https://doi.org/10. 1007/s11695-018-3484-5.

5. Mehta P, McAuley DF, Brown M, et al. COVID-19: consider cytokine storm syndromes and immunosuppression. Lancet. 2020.

6. Barazzoni R, Bischoff SC, Breda J, et al. ESPEN expert statements and practical guidance for nutritional management of individuals with SARS-CoV-2 infection I ESPEN-ovo stručno mišljenje i praktične smjernice za nutritivnu potporu bolesnika s infekcijom SARS-CoV-2. Lijec Vjesn. 2020.

7. Lechien JR, Chiesa-Estomba CM, De Siati DR, et al. Olfactory and gustatory dysfunctions as a clinical presentation of mildto-moderate forms of the coronavirus disease (COVID-19): a multicenter European study. Eur Arch Oto-Rhino-Laryngology. 2020;277:13. https://doi.org/10.1007/s00405-020-05965-1.

8. Briguglio M, Pregliasco FE, Lombardi G, et al. The malnutritional status of the host as a virulence factor for new coronavirus SARSCoV-2. Front Med. 2020. https://doi.org/10.3389/fmed.2020.00146.

9. Di Filippo L, De Lorenzo R, D’Amico M, et al. COVID-19 is associated with clinically significant weight loss and risk of malnutrition, independent of hospitalisation: a post-hoc analysis of a prospective cohort study. Clin Nutr. 2020. https://doi.org/10. 1016/j.clnu.2020.10.043.

10. Crook H, Raza S, Nowell J, et al. Long COVID-mechanisms, risk factors, and management. BMJ. 2021;374:n1648. https:// doi.org/10.1136/bmj.n1648.

11. National Institute for Health and Care Excellence, Practitioners RC of G, Scotland HI. COVID-19 rapid guideline : managing the long-term effects of COVID-19. NICE Guidelines. 2020.
12. Salamanna F, Veronesi F, Martini L, et al. Post-COVID-19 syndrome: the persistent symptoms at the post-viral stage of the disease. A systematic review of the current data. Front Med. 2021.

13. Maciejewski ML, Arterburn DE, Van Scoyoc L, et al. Bariatric surgery and long-term durability of weight loss. JAMA Surg. 2016. https://doi.org/10.1001/jamasurg.2016.2317.

14. Odone A, Delmonte D, Scognamiglio T, Signorelli C. COVID-19 deaths in Lombardy, Italy: data in context. Lancet Public Health. 2020.

15. World Health Organization. Public health surveillance for COVID-19: Interim Guidance. 2020.

16. Marchesi F, Valente M, Riccò M, et al. Effects of bariatric surgery on COVID-19: a multicentric study from a high incidence area. Obes Surg. 2021. https://doi.org/10.1007/s11695-020-05193-w.

17. Li G, Chen Z, Lv Z, et al. Diabetes mellitus and COVID-19: associations and possible mechanisms. Int J Endocrinol. 2021.

18. Zhang P, He Z, Yu G, et al. The modified NUTRIC score can be used for nutritional risk assessment as well as prognosis prediction in critically ill COVID-19 patients. Clin Nutr. 2021. https://doi. org/10.1016/j.clnu.2020.05.051.

19. Li T, Zhang Y, Gong C, et al. Prevalence of malnutrition and analysis of related factors in elderly patients with COVID-19 in Wuhan, China. Eur J Clin Nutr. 2020. https://doi.org/10.1038/ s41430-020-0642-3.

20. Arentson-Lantz EJ, English KL, Paddon-Jones D, Fry CS. Fourteen days of bed rest induces a decline in satellite cell content and robust atrophy of skeletal muscle fibers in middle-aged adults. $\mathbf{J}$ Appl Physiol. 2016. https://doi.org/10.1152/japplphysiol.00799. 2015.

21. Kortebein P, Symons TB, Ferrando A, et al. Functional impact of 10 days of bed rest in healthy older adults. J Gerontol - Ser A Biol Sci Med Sci. 2008. https://doi.org/10.1093/gerona/63.10.1076.

22. English KL, Mettler JA, Ellison JB, et al. Leucine partially protects muscle mass and function during bed rest in middle-aged adults. Am J Clin Nutr. 2016. https://doi.org/10.3945/ajen.115.112359.

23. Fearon K, Strasser F, Anker SD, et al. Definition and classification of cancer cachexia: an international consensus. Lancet Oncol. 2011.

24. Varela ML, Mogildea M, Moreno I, Lopes A. Acute inflammation and metabolism. Inflammation. 2018.

25. Burfeind KG, Michaelis KA, Marks DL. The central role of hypothalamic inflammation in the acute illness response and cachexia. Semin Cell Dev Biol. 2016.

26. Cederholm T, Jensen GL, Correia MITD, et al. GLIM criteria for the diagnosis of malnutrition - a consensus report from the global clinical nutrition community. Clin Nutr. 2019. https://doi.org/10. 1016/j.clnu.2018.08.002.

27. Hawkins RB, Raymond SL, Stortz JA, et al. Chronic critical illness and the persistent inflammation, immunosuppression, and catabolism syndrome. Front Immunol. 2018. https://doi.org/10. 3389/fimmu.2018.01511.

28. Office for National Statistics. Prevalence of ongoing symptoms following coronavirus ( COVID-19) infection in the UK. In: Off Natl Stat. 2021.

29. Otte MS, Klussmann JP, Luers JC. Persisting olfactory dysfunction in patients after recovering from COVID-19. J Infect. 2020.

30. Fjaeldstad AW. Prolonged complaint s of chemosensory loss after COVID-19. Dan Med J. 2020.

31. Paderno A, Mattavelli D, Rampinelli V, et al. Olfactory and gustatory outcomes in COVID-19: a prospective evaluation in nonhospitalized subjects. Otolaryngol - Head Neck Surg (United States). 2020. https://doi.org/10.1177/0194599820939538.

32. Short KR, Kedzierska K, van de Sandt CE. Back to the future: lessons learned from the 1918 influenza pandemic. Front Cell Infect Microbiol. 2018. https://doi.org/10.3389/fcimb.2018.00343. 
33. Bel Lassen P, Poitou C, Genser L, et al. COVID-19 and its severity in bariatric surgery-operated patients. Obesity. 2021. https://doi. org/10.1002/oby.23026.

34. Földi M, Farkas N, Kiss S, et al. Obesity is a risk factor for developing critical condition in COVID-19 patients: a systematic review and meta-analysis. Obes Rev. 2020.

35. Chang TH, Chou CC, Chang LY. Effect of obesity and body mass index on coronavirus disease 2019 severity: a systematic review and meta-analysis. Obes Rev. 2020. https://doi.org/10.1111/obr. 13089.
36. Aminian A, Fathalizadeh A, Tu C, et al. Association of prior metabolic and bariatric surgery with severity of coronavirus disease 2019 (COVID-19) in patients with obesity. Surg Obes Relat Dis. 2021. https://doi.org/10.1016/j.soard.2020.10.026.

Publisher's Note Springer Nature remains neutral with regard to jurisdictional claims in published maps and institutional affiliations. 